\title{
Hormone replacement therapy: the risks and benefits of treatment
}

\author{
${ }^{1} \mathrm{C}$ Hardie, ${ }^{2} \mathrm{C}$ Bain, ${ }^{3} \mathrm{M}$ Walters \\ ${ }^{1}$ Specialist Registrar in Obstetrics and Gynaecology; ${ }^{2}$ Consultant Obstetrician and Gynaecologist, Glasgow Royal Infirmary; ${ }^{3}$ Reader in \\ Medicine and Honorary Consultant Physician, Division of Cardiovascular and Medical Sciences, University of Glasgow, UK
}

ABSTRACT Several recent large studies have provoked concern among both health professionals and the general public regarding the safety of hormone replacement therapy (HRT). This article provides a review of the current literature surrounding the risks and benefits of HRT in postmenopausal women, and how the data can be applied safely in everyday clinical practice.

KEYWORDS Hormone replacement therapy, menopause, oestrogen

DECLARATION OF INTERESTS No conflict of interests declared.
Published online July 2009

Correspondence to $M$ Walters, Faculty of Medicine, University of Glasgow, Glasgow GI 2 8QQ, UK

tel. +44 (0)14I 3305921

e-mail

m.walters@clinmed.gla.ac.uk
Worldwide, approximately 47 million women will undergo the menopause every year for the next 20 years.' The lack of circulating oestrogens which occurs during the transition to menopause presents a variety of symptoms, including hot flushes, night sweats, mood disturbance and vaginal atrophy, and these can be distressing in almost $50 \%$ of women.

For many years, oestrogen alone or in combination with progestogens, otherwise known as hormone replacement therapy (HRT), has been the treatment of choice for control of problematic menopausal symptoms and for the prevention of osteoporosis. However, the use of HRT declined worldwide following the publication of the first data from the Women's Health Initiative (WHI) trial in 2002. ${ }^{2}$ The results led to a surge in media interest surrounding HRT usage, with the revelation that there was an increased risk of breast cancer and, contrary to expectation, coronary heart disease (CHD) in those postmenopausal women taking oestrogen plus progestogen HRT. Following this, both the Heart and Estrogen/Progestin Replacement Study Follow-up (HERS II) (3,4 $^{3}$ and the Million Women Study ${ }^{5}$ published results that further reduced enthusiasm for HRT use, showing increased risks of breast cancer $^{5}$ and venous thromboembolism (VTE), ${ }^{4}$ and the absence of previously suggested cardioprotective effects ${ }^{3}$ in HRT users. The resulting fear of CHD and breast cancer in HRT users left many women with menopausal symptoms and few effective treatment options.

Continued analysis of data relating to these studies has been aimed at understanding whether or not the risks associated with HRT are, in fact, limited to a subset of women. A recent publication from the International Menopause Society ${ }^{6}$ has stated that HRT remains the first-line and most effective treatment for menopausal symptoms. In this article we examine the evidence that has contributed to common perceptions among health professionals and women alike, and clarify the balance of risk and benefit to be considered by women using HRT.

\section{INITIAL INTERPRETATION OF THE WHI TRIAL}

The US-led WHI trial assessed the potential benefit of oral HRT $(0.625 \mathrm{mg}$ conjugated equine oestrogen and $2.5 \mathrm{mg}$ medroxyprogesterone acetate daily) in a multicentre, randomised placebo-controlled trial (RCT) with more than 8,000 women in each arm. It is important to emphasise that this huge RCT was never designed to examine the benefits or risks of HRT when used for the management of menopause-associated vasomotor symptoms. The mean age of enrolment in the trial was 63 years, which is older than the typical age of women seeking HRT preparations in the UK.

Using a global index to examine the risks and benefits of hormone usage, the initial results from the WHI demonstrated that the risks of long-term HRT were greater than the benefits, which led to premature closure of the study. These elevated risks encompassed $\mathrm{CHD}$, breast cancer, stroke and VTE in postmenopausal women using HRT. Importantly, a quality of life analysis was not included. Due to concern over the initial results published by the WHI in 2002, many health professionals and patients considered the use of such hormones as 'unsafe', leading to a $50 \%$ reduction in HRT prescribing.'

The main issue was that results were extrapolated from an older population of women than those who would normally be seeking HRT for the management of menopausal symptoms. Subsequent subgroup analysis of the data suggested that age and years from start of menopause are major determinants of the risk-benefit balance of HRT, and that there may be advantages in using appropriately timed HRT in younger women. ${ }^{8,9}$ Although these subgroup analyses were conducted post hoc and lack power, it remains evident that in younger women commencing HRT (aged 50-59 years) the WHI global index is actually in favour of HRT usage. 


\section{CARDIOVASCULAR EFFECTS OF HRT REVISITED}

One of the key messages from the WHI in 2002 was that HRT should not be prescribed to prevent age-related chronic disease, in particular CHD. This was contradictory to previous advice based on observational studies. However, recent subgroup analysis has shown that in healthy individuals using HRT in the early postmenopausal years (age 50-59 years), there was no increased CHD risk and HRT may potentially have a cardioprotective effect. $^{8}$ Recent WHI data have suggested that oestrogenalone HRT in compliant women under 60 years of age delays the progression of atheromatous disease (as assessed by coronary arterial calcification). ${ }^{9}$ The Nurses Health Study, a large observational study in the USA, demonstrated that the increase in stroke risk appeared to be modest in younger women, with no significant increase if HRT had been used for less than five years. There was also evidence that the stroke risk conferred by HRT appears to be dose-related..$^{10}$

\section{BONE EFFECTS OF HRT}

Hormone replacement therapy is associated with beneficial effects on bone mineral density, the prevention of osteoporosis and an improvement in osteoarthritic symptoms. The WHI clearly demonstrated that HRT was effective in the prevention of all fractures secondary to osteoporosis. " The downturn in HRT prescribing, related to the concern regarding vascular and breast cancer risks, is expected to cause an increase in fracture risk, and it is predicted that in the USA there will be a possible excess of $\geq 43,000$ fractures per year in the near future."

\section{CANCER RISKS RELATED TO HRT}

\section{Breast cancer}

There is a common perception that all HRT preparations cause breast cancer. Observational studies and the WHI have shown that there is an increase in breast cancer risk over five years using combined HRT of about eight extra cases per 10,000 women per year., ${ }^{5} 13$ The oestrogen-only arm did not show an increase in breast cancer incidence within the seven years of follow-up of the WHI. ${ }^{14}$ This finding conflicts with observational data from the Million Women Study, ${ }^{5}$ which has reported an increased risk of incident breast cancer in users of oestrogen-only and combined HRT, albeit with a lesser increase in risk in the former group. Interestingly, the sharp decline in HRT use over recent years has correlated with a decline in breast cancer incidence in the USA, but not so clearly elsewhere. This decline was noted before the WHI trial was published and may be coincidental.

\section{Ovarian cancer}

The UK Million Women Study concluded that HRT taken over five years resulted in one extra ovarian cancer in approximately 2,500 HRT users, and one extra ovarian death in roughly 3,300 users. ${ }^{15}$ Prospective randomised controlled trials have suggested a modest effect on the risk of developing ovarian cancer in HRT users, but these studies have not demonstrated an increased risk of death. ${ }^{16}$

\section{HRT AND VENOUS THROMBOEMBOLISM}

Until recent years, the exogenous oestrogens used in HRT were not considered to increase the risk of VTE. Although the absolute risk of VTE with HRT remains low, most studies have shown the risk to be at least two-fold higher with standard doses of oral HRT versus placebo. ${ }^{17}$

The risk of venous thrombosis is highest in the first year of use, and any underlying thrombophilia has been shown to increase the risk of VTE. ${ }^{18}$ The absolute risk of VTE is low in healthy postmenopausal women, but the use of HRT should be considered very carefully in women with known thrombophilic tendencies and may be best avoided in women with a previous history of VTE. The transdermal route is thought to have less effect on clotting factors ${ }^{19}$ and may be associated with a lower risk of thrombotic events, ${ }^{19}$ but more studies are needed.

\section{QUALITY OF LIFE ISSUES SURROUNDING HRT}

The understanding of the menopause and its effect on quality of life is interpreted variably worldwide. ${ }^{6}$ The WHI quality of life analysis lacked power to draw any sufficient conclusions. ${ }^{20}$ However, the WISDOM study ${ }^{21}$ has shown a measurable improvement in quality of life, sleep, sexuality and joint pain in symptomatic women by the administration of oestrogen HRT.

\section{CONCLUSIONS}

The WHI results published in 2002 led to a significant decline in patient and clinician confidence in the use of HRT. Further analysis of the data has prompted a re-evaluation of this initial reaction, and recognition that many women may have been 'denied' treatment. Now is the time to responsibly restore confidence regarding the benefit of HRT in the treatment of menopausal symptoms when used judiciously. Hormone replacement therapy is undoubtedly effective in the treatment of vasomotor symptoms, and confers protection against osteoporotic fractures. The oncological risks are relatively well characterised and patients considering HRT should be made aware of these. The cardiovascular risk of HRT in younger women without overt vascular disease is less well defined and further work is required to address this important question. In the interim, decisions regarding HRT use should be made on a case-by-case basis following informed discussion of the balance of risk and benefit. The lowest dose of hormone necessary to alleviate menopausal symptoms should be used, and the prescription reviewed on a regular basis. 


\section{KEY POINTS}

- The menopause causes troublesome symptoms in almost $50 \%$ of women.

- There has been a significant decline in the prescribing of hormone replacement therapy (HRT) since the publication of the first data from the Women's Health Initiative trial in 2002, which suggested that HRT had an adverse effect on vascular risk and increased the rate of breast cancer.

- Recent data suggest that age and years from the start of menopause are major determinants of the riskbenefit balance of HRT and that there may be advantages in using appropriately timed HRT in younger women. Hormone replacement therapy used in the early menopausal years may potentially have a cardioprotective effect.

- Hormone replacement therapy is effective in the prevention of osteoporotic fractures, but due to decreased prescribing following publication of the WHI trial it is predicted that preventable fractures could increase in coming years.

- Both randomised trials and observational studies have suggested a modest increase in the risk of breast and ovarian cancer in HRT users.

\section{REFERENCES}

I Hill K. The demography of menopause. Maturitas 1996; 23:I13-27. doi:10.1016/0378-5I22(95)00968-X

2 Writing Group for the Women's Health Initiative Investigators Risks and benefits of estrogen plus progestin in healthy postmenopausal women: principal results from the Women's Health Initiative randomized controlled trial. JAMA 2002; 288:32I-33. doi:10.100I/jama.288.3.321

3 Grady D, Herrington D, Bittner $V$ et al., for the HERS Research Group. Cardiovascular disease outcomes during 6.8 years of hormone therapy: Heart and Estrogen/progestin Replacement Study follow-up (HERS II). JAMA 2002; 288:49-57. doi:10.100I/ jama.288.1.49

4 Hulley S, Purberg C, Barrett-Connor E et al., for the HERS Research Group. Noncardiovascular disease outcomes during 6.8 years of hormone therapy: Heart and Estrogen/progestin Replacement Study follow-up (HERS II). JAMA 2002; 288:58-66. doi:10.100I/jama.288.1.58

5 Beral V, Million Women Study Collaborators. Breast cancer and hormone replacement therapy in the Million Women Study. Lancet 2003; 362:419-27. doi:I0.1016/S0I40-6736(03)14596-5

6 Pines A, Sturdee W, Birkhauser MH et al., for the International Menopause Society. HRT in the early menopause: scientific evidence and common perceptions. Climacteric 2008; II:267-72. doi: I0.1080/I3697/ 30802226866

7 Kim N, Gross C, Curtis J et al.The impact of clinical trials on the use of hormone replacement therapy: a population-based study. J Gen Intern Med 2005; 20:1026-3I. doi:I0.IIII/j.1525-I497.2005.022I.x

8 Rossouw JE, Prentice RL, Manson JE et al. Postmenopausal hormone therapy and risk of cardiovascular disease by age and years since menopause. JAMA 2007; 297:1465-77. doi:10.1001/ jama.297.13.1465

9 Manson JE, Allison MA, Rossouw JE et al. Estrogen therapy and coronary-artery calcification. N Engl J Med 2007; 356:259I-602. doi:I0.1056/NEJMoa07I5I3

10 Grodstein F, Manson JE, Stampfer J et al. Postmenopausal hormone therapy and stroke. The role of time since menopause and age of initiation of hormone therapy. Arch Intern Med 2008; 168:86I-6. doi:I0.100I/archinte.168.8.86I
II Jackson RD, Wactawski-Wende J, LaCroiz AZ et al. Effects of conjugated equine estrogen on risk of fractures and $B M D$ in postmenopausal women with hysterectomy: results from the Women's Health Initiative randomized trial.J Bone Miner Res 2006; 21:817-28. doi:10.1359/jbmr.0603/2

12 Gambacciani M, Ciaponi M, Genazzani AR. The HRT misuse and osteoporosis epidemic: a possible future scenario. Climacteric 2007; 10:273-5. doi: 10. I080//3697/3070 I5 I I 277

13 Chlebowski RT, Hendrix SL, Langer RD et al. Influence of estrogen plus progestin on breast cancer and mammography in healthy postmenopausal women: the Women's Health Initiative randomized trial.JAMA 2003; 289:3243-53. doi:10.1001/jama.289.24.3243

14 Stefanick ML, Anderson GL, Margolis KL et al. Effects of conjugated equine estrogens on breast cancer and mammography screening in postmenopausal women with hysterectomy. JAMA 2006; 295:1647-57. doi:10.1001/jama.295.14.1647

15 Million Women Study Collaborators. Ovarian cancer and hormone replacement therapy in the Million Women Study. Lancet 2007; 369:1703-10. doi:10.1016/S0140-6736(07)60534-0

16 Anderson GL, Judd HL, Kaunitz AM et al. Effects of estrogen plus progestin on gynecologic cancers and associated diagnostic procedures. JAMA 2003; 290: 1739-48. doi:10.100I/jama.290.13.1739

17 Cushman M, Kuller LH, Prentice et al. Estrogen plus progestin and risk of venous thrombosis. JAMA 2004; 292:1573-80. doi:10.1001/ jama.292.13.1573

18 Canonico M, Oger E, Plu-Bureau G et al. Hormone therapy and venous thromboembolism among postmenopausal women: impact of the route of estrogen administration and progestogens: the ESTHER study. Circulation 2007; 1|5:840-5. doi:10.1I6I/ CIRCULATIONAHA. 106.642280

19 Scarabin PY, Oger E, Plu-Bureau G et al. Differential association of oral and transdermal oestrogen-replacement therapy with venous thromboembolism risk. Lancet 2003; 362:428-32. doi:10.1016/ SOI40-6736(03) I 4066-4

20 Hays J, Ockene JK, Brunner RL et al. Effects of estrogen plus progestin on health-related quality of life. N Engl J Med 2003; 348: 1839-54. doi: I0.1056/NEJMoa0303II

21 Welton AJ, Vickers MR, Kim J et al. Health related quality of life after combined hormone replacement therapy: randomised controlled trial. BMJ 2008; 337:al I90. doi:I0.1 I36/bmj.al 190 